Original research article

\title{
A comparative analysis of lipid content and fatty acid composition in muscle, liver and gonads of Seriola fasciata Bloch 1793 based on gender and maturation stage
}

\author{
Natacha Nogueira, ${ }^{\mathrm{a}, *}$, Igor Fernandes ${ }^{\mathrm{b}}$, Tomásia Fernandes ${ }^{\mathrm{b}}$, Nereida Cordeiro ${ }^{\mathrm{b}}$ \\ a Mariculture Center of Calheta, Vila da Calheta, 9370-133 Calheta, Madeira, Portugal \\ ${ }^{\mathrm{b}}$ University of Madeira, Faculty of Exact Sciences and Engineering, 9000-390 Funchal, Madeira, Portugal
}

\section{A R T I C L E I N F O}

\section{Article history:}

Received 28 June 2016

Received in revised form 24 October 2016

Accepted 10 November 2016

Available online 13 November 2016

\section{Keywords:}

Food analysis

Seriola fasciata

Lesser amberjack

Maturation stage

Gender

Lipid content

Fatty acid profile

Food composition

Nutritional values

\begin{abstract}
A B S T R A C T
The fat content and fatty acid profile of commercially important Seriola fasciata (Lesser amberjack) was determined in samples of muscle, liver and gonads of both genders at pre-spawning and spawning stage. Results indicated that edible muscle of pre-spawning fish had high fat content $(>8 \%)$, lowering to medium fat (4-8\%) content during spawning stage. Livers and gonads presented more constant and relatively high lipid contents. Saturated fatty acids constituted the majority of the fatty acids, followed by monounsaturated fatty acids, with palmitic acid and oleic acid as the major fatty acid in all tissues. EPA and DHA content increased in spawning females and highest amounts were observed in the ovaries (222 and $1440 \mathrm{mg} / 100 \mathrm{~g}$ respectively). Livers presented lowest PUFAs content regardless of maturity stage and $\Sigma n-6 / \Sigma n-3$ ratio was influenced by gender in this organ. $\Sigma n-3 / \Sigma n-6$ was gender related during pre-spawning stage, increasing in spawning maturation stage. Results highlight variations in the nutritional value of the different tissues according to maturity stage and gender, emphasizing the need for consumer awareness of these changes.
\end{abstract}

(c) 2016 Published by Elsevier Inc.

\section{Introduction}

Foods from the aquatic environment are a complete and unique source of both macro- and micronutrients required in a healthy diet. Fatty acids (FAs), especially the ones in fish lipids, are very important nutritional elements for human health and have been associated with the prevention of several diseases (Simopoulos, 2000). Although their content is species specific, from a human consumption perspective, the polyunsaturated fatty acids (PUFAs) composition in fish strongly determines their nutritional quality, since fish are key in supplying $n-3$ PUFAs (Steffens, 1997). In particular, $n-3$ fatty acids eicosapentaenoic acid (EPA, 20:5n-3) and docosahexaenoic acid (DHA, 22:6n-3) display several properties beneficial for human health (Prato and Biandolino, 2012) and have been linked to the prevention of cardiovascular diseases (Sidhu, 2003) and cancers, reduction of the risk of coronary heart disease, decreasing of mild hypertension, lowering the incidence of diabetes and possible alleviation of the symptoms of rheumatoid arthritis (Simopoulos, 2002). Still, actual dietary

\footnotetext{
* Corresponding author.

E-mail address: natachacnogueira@gmail.pt (N. Nogueira).
}

intakes in the western diet tends to be too low in $n-3$ fatty acids, mainly due to the low consumption of seafood and a lack of adequate information for consumers regarding the nutritional value of these foodstuffs (Prato and Biandolino, 2012).

The total lipid and fatty acid composition of fish in different tissues varies according to several parameters, such as fishing season, geographical location, size, sex, and reproductive cycle period (Luzia et al., 2003). While some fish (e.g., salmon, trout and herring) store FAs predominantly in muscle tissues, lean fish accumulate the FAs in body cavities and perivisceral organs (e.g., liver and gonads) (Guil-Guerrero et al., 2011). Still, the liver and gonads are usually discarded when fish are prepared for human consumption, thus wasting a potential source of PUFAs. Knowing that there is a growing demand for PUFAs, the characterization of suitable new sources from discarded by-products would be desirable (Jayasinghe et al., 2003), not only for increasing the profitability of the by-products of the fishing industry, but also for the possibility of finding PUFA-oils with higher quality than traditional ones.

Of the nine valid Seriola species that occur worldwide, greater amberjack ( $S$. dumerilli) is the most commercialized because it is distributed worldwide, offers excellent flesh quality, high market price and high growth rates in the wild and in captivity (Jerez et al., 
2006). The lesser amberjack (S. fasciata), a closely related species, is also a coastal or demersal carangid that exists in the western Atlantic (USA, Gulf of Mexico, Cuba, Puerto Rico and Bermuda) and in the eastern Atlantic (Balearic Islands; Sicily; Azores; Galiza) (Machado and Barreiros, 2006). This is a valuable fishery resource whose increased availability (Andaloro et al., 2005; Deidun et al., 2011; Kapiris et al., 2014; Jawad et al., 2015) enhances its fisheries potential.

Most published work on S. fasciata focuses on the species distribution and on the occurrence of human food-borne intoxication caused by ingestion of S. fasciata contaminated with ciguatoxins (Otero et al., 2010). To our best knowledge, only Nogueira et al. (2013) reported data on lipid and fatty acid composition of this species.

This work aimed to determine lipids and FA of muscle, liver and gonads of lesser amberjack as a further contribution to the knowledge of valuable alternative edible sources of PUFAs in sexually mature fish.

\section{Material and methods}

\subsection{Fish samples}

Wild fish ( $n=22)$ were obtained from local fishermen between the period of August and September of 2012. Fish were caught near Selvagens and Desertas islands (Portugal), Eastern Atlantic and kept on ice until laboratory arrival. Within the first $36 \mathrm{~h}$ after being caught fish were examined for individual meristic identification and morphometric data were collected according to Smith-Vaniz (1986) and Smith-Vaniz (2002). Sampled fish were then dissected to determine sex and gonad maturity stage using macroscopic and microscopic examination. Maturation stage was determined using a five stage scale: I- immature; II- recovering; III-pre-spawning (developing); IV- spawning, V-spent, adapted from BrownPeterson et al. (2011).

Whole gonads and livers were examined and weighted to the nearest $0.01 \mathrm{~g}$. The gonadosomatic index (GSI) and hepatosomatic index (HSI), which are common metrics of reproductive allocation and reproductive condition in fisheries biology, were calculated as:

(gonad or liver mass/body mass) $\times 100 \%$

The muscle tissue from the dorsal side that is directly under the dorsal fin and above the lateral line was removed (approximately $3 \times 3 \mathrm{~cm}$ sample). Samples of dorsal muscle, whole liver and gonads were kept at $-20^{\circ} \mathrm{C}$ for a period no longer than three months. Prior to lipid content and fatty acid determination, samples were homogenized and pooled according to gender and maturity stage. All pooled samples were then freeze-dried at $-60{ }^{\circ} \mathrm{C}$ and $0.1 \mathrm{mbar}$ in a Savant freeze-dryer $(n=4)$. Samples were considered dried when the residual water content was less than
0.4\% (w/w), using a Gibertini Eurotherm electronic moisture balance (Gibertini Elettronica, Novate Milanese MI, Italy).

\subsection{Chemicals}

Methanol, BHT, heptane, as well as the standards samples used were supplied by Sigma Aldrich (St. Louis MO, United States). Chloroform and potassium chloride were supplied by VWR (Radnor PA, United States), while acetyl chloride was supplied by Merck (Darmstadt, Germany). All of the aforementioned chemicals used in this work were of analytical grade (99\% purity).

\subsection{Lipid and fatty acid analysis}

Extraction of total lipid was performed according to modified Bligh and Dyer (1959), as described in Nogueira et al. (2013). Around $100 \mathrm{mg}$ of lyophilized sample were weighed and $3 \mathrm{~mL}$ of a solution containing methanol/chloroform/BHT (2:1:0.01\%) and $400 \mu \mathrm{L}$ of saturated solution of potassium chloride were added. After homogenization, $2 \mathrm{~mL}$ of chloroform and $2 \mathrm{~mL}$ of distilled water were added, and placed under agitation for $20 \mathrm{~min}$. The organic phase was collected and the solvent removed using a rotary evaporator at $40^{\circ} \mathrm{C}$. The lipid content was determined gravimetrically after drying in the desiccator. The samples were stored at $-20^{\circ} \mathrm{C}$ until further analysis. Determinations were made in triplicate and the results are expressed in $\mathrm{g}$ of lipids/100 $\mathrm{g}$ sample.

\subsubsection{Fatty acid analysis}

Total lipid extracts were analysed for their fatty acid composition as fatty acid methyl esters (FAMEs) as described in Nogueira et al. (2013). Briefly, the fatty acids were converted to FAMEs by adding a mixture of acetyl chloride and methanol $(1: 19 \mathrm{v} / \mathrm{v})$ to total lipid aliquots that were heated at $80^{\circ} \mathrm{C}$ for $1 \mathrm{~h}$.

FAMEs were analysed by gas chromatography (Agilent HP 6890) equipped with a mass selective detector (Agilent 5973) and a fused silica capillary column Supelcowax ${ }^{\mathrm{TM}} 10(30 \mathrm{~m} \times 0.25 \mathrm{~mm}$ inner diameter, $0.25 \mu \mathrm{m}$ film thickness) from Supelco (Bellefonte, PA). The chromatographic conditions were: initial temperature, $40^{\circ} \mathrm{C}$ for $5 \mathrm{~min}$; temperature gradient, $2^{\circ} \mathrm{C} \mathrm{min}^{-1}$; final temperature, $250{ }^{\circ} \mathrm{C}$ for $5 \mathrm{~min}$; injector temperature, $260^{\circ} \mathrm{C}$; transfer-line temperature, $260^{\circ} \mathrm{C}$; split ratio, $1: 100$. Helium was used as the carrier gas with a flow of $1.0 \mathrm{~mL} \mathrm{~min}^{-1}$.

FAMEs identification was made by comparison of the retention times and mass spectra to those of known standards (bacterial acid methyl esters CP mix; PUFA No. 3, from menhaden oil; Supelco 37 component FAME mix). Quantification of FAs in fish samples was made according to the response factor determined for each FA in the standards in relation to the internal standard (21:0, heneicosanoic acid). Determinations were performed in quadruplicate for

Table 1

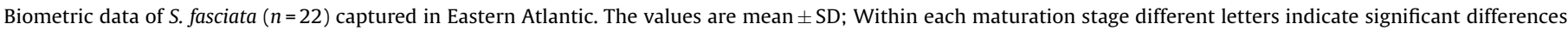
between genders $(p<0.05)$ for each of the biometric characteristics.

\begin{tabular}{|c|c|c|c|c|}
\hline \multirow[t]{3}{*}{ Morphometry } & \multicolumn{4}{|l|}{ Maturation Stage } \\
\hline & \multicolumn{2}{|c|}{ Pre-Spawning (stage III) } & \multicolumn{2}{|c|}{ Spawning (stage IV) } \\
\hline & Female $(n=6)$ & Male $(n=5)$ & Female $(n=5)$ & Male $(n=6)$ \\
\hline Body weight (g) & $3179.7 \pm 784.5^{\mathrm{a}}$ & $4270.5 \pm 590.4^{\mathrm{a}}$ & $4342.6 \pm 1200.0^{\mathrm{a}}$ & $6929.8 \pm 2060.8^{b}$ \\
\hline Fork length (mm) & $552.5 \pm 56.4^{\mathrm{a}}$ & $620.0 \pm 28.3^{\mathrm{a}}$ & $621.8 \pm 84.1^{\mathrm{a}}$ & $699.5 \pm 87.0^{\mathrm{a}}$ \\
\hline Body height (mm) & $149.7 \pm 14.3^{\mathrm{a}}$ & $176.0 \pm 7.0^{\mathrm{a}}$ & $166.3 \pm 13.7^{\mathrm{a}}$ & $193.5 \pm 17.2^{\mathrm{b}}$ \\
\hline Body depth (mm) & $116.0 \pm 43.1^{\mathrm{a}}$ & $83.0 \pm 2.8^{\mathrm{a}}$ & $77.0 \pm 11.3^{\mathrm{a}}$ & $96.50 \pm 14.1^{\mathrm{a}}$ \\
\hline Gonadosomatic index & $1.1 \pm 0.3^{\mathrm{a}}$ & $3.8 \pm 0.4^{\mathrm{b}}$ & $1.3 \pm 0.5^{\mathrm{a}}$ & $0.64 \pm 0.2^{\mathrm{b}}$ \\
\hline Hepatosomatic index & $1.9 \pm 0.7^{\mathrm{a}}$ & $0.7 \pm 0.2^{\mathrm{a}}$ & $1.1 \pm 0.6^{\mathrm{a}}$ & $1.0 \pm 0.3^{b}$ \\
\hline
\end{tabular}


each pooled sample and the results were expressed in $\mathrm{mg} / 100 \mathrm{~g}$. The chemicals were all of analytical grade and used as received.

\subsection{Statistical analysis}

Data are reported as mean $\pm S D$ and analyzed by one-way analysis of variance (ANOVA) to determine differences between means at $5 \%$ confidence level. All variables were checked for normality and homogeneity of variance, using the KolmogorovSmirnoff and the Levene tests, respectively. All statistical analyses were performed using SPSS. v 23 for Windows (SPSS Inc., Chicago, IL).

\section{Results and discussion}

Fish sexual maturation stage and biological parameters are presented in Table 1. Morphometric data showed that males presented greater mean body weight and height than females. It is worth noticing that mean fork length registered for males on maturation stage IV, with an absolute maximum of $761 \mathrm{~mm}$, was bigger than the $680 \mathrm{~mm}$ referenced for this species by Smith-Vaniz et al. (2015).

Lipids are the major metabolic energy resource involved in the reproductive process in fishes (Tocher, 2003). Lipid content (TL) was gender dependent in dorsal muscle and gonads of prespawning fish (Fig. 1), with females presenting significantly higher TL than males at this maturity stage $(p<0.05)$. Liver presented overall highest lipid content (Fig. 1 ), with a maximum value observed in pre-spawning males $(27 \mathrm{~g} / 100 \mathrm{~g})$, indicating that this species tends to accumulate lipids in liver. These values seem to fit the average levels for lipids of several marine fish species (GuilGuerrero et al., 2011), which can offer a potential source of valuable fats. As the liver plays an important role in fish gonad development, being responsible for large lipid and fatty acid deposits in the ovary, it was not surprisingly that ovaries and testes also presented high TL at both maturity stages, with significant differences between gender $(p<0.05)$. From a nutritional standpoint, fillets (muscle) of lesser amberjack at pre-spawning stage can be placed amongst those fish with high-fat content $(>8 \%)$, but fish at spawning stage are considered medium-fat (4-8\%) (Ackman, 1989), which is in agreement with what has been found for other Seriola species (Thakur et al., 2009; Rodriguez-Barreto et al., 2012; Saito, 2012).

Fatty acid profile revealed that saturated fatty acids (SFAs) constituted the majority of the fatty acids pool followed by monounsaturated fatty acids (MUFAs) and polyunsaturated fatty

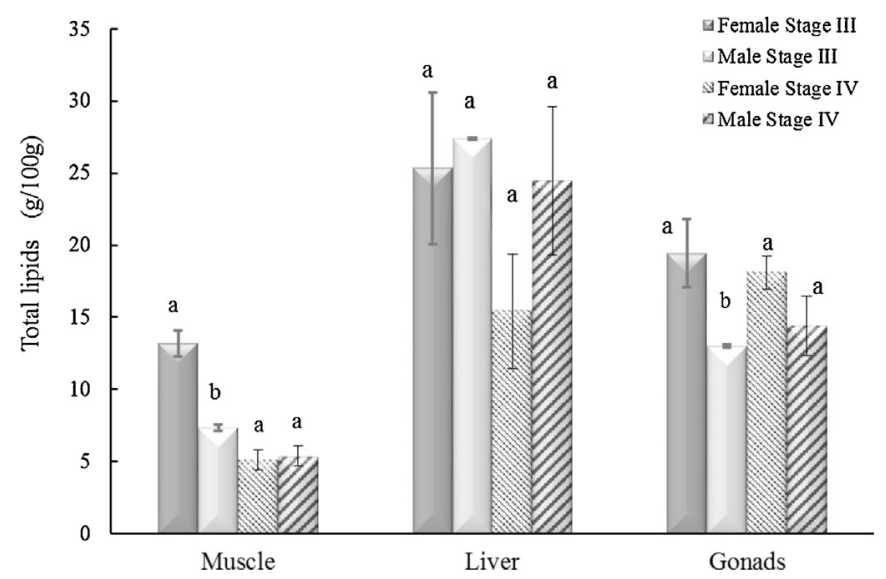

Fig. 1. Total lipid content $(\mathrm{g} / 100 \mathrm{~g})$ in dorsal muscle, whole liver and gonad of $S$ fasciata females and males at pre-spawning and spawning stage. The values are mean of three replicates $\pm S D$; within each maturation stage different letters indicate significant differences between genders $(p<0.05)$. acids (PUFAs) (Tables 2 and 3). The observed amounts of SFAs and MUFAs which are typical of many fish species (Turchini et al., 2003; Grigorakis, 2007), particularly in fish from warm or temperate regions (Guil-Guerrero et al., 2011), can be explained by the fact that SFAs and MUFAs are storage lipids that can preferentially be used as an energy source.

Palmitic acid (C16:0), the most prevalent SFA in the human diet, was noticed as the major SFAs and higher amounts of this fatty acid were detected in liver samples, followed by gonads and muscle. The dominance of palmitic acid in fish lipid has also been reported by other authors (Osman et al., 2001; Guil-Guerrero et al., 2011), since this FA is documented as a predominant source of potential metabolic energy during fish growth (Henderson et al., 1984).

From a nutrition standpoint, MUFAs have received increasing attention because of their mixed effects on human health. Recent evidence tends to indicate more beneficial effects, in particular, on reducing risk of cardiovascular diseases and other inflammationrelated diseases (Mashek and Wu, 2015). As a source of MUFAs, liver samples of both genders proved to be a better source of these FAs, especially during pre-spawning stage. Amongst this FA family, oleic acid (OA, C18:1n-9) and palmitoleic acid (PA, C16:1n-9) are the most abundant MUFA in the human body (Miyazaki et al., 2001). OA is also the prominent FAa among MUFAs in the lipids of many marine fish, so it was not unexpected to find that this FA was the major MUFA in all $S$. fasciata samples. Additionally, though a marked reduction was observed between pre-spawning and spawning fish, there seems to exist a gender effect, as female values for OA and PA where higher than for males.

Recently, there has been increased interest in the role of dietary intake of fish-derived $n-3$ fatty acids, namely eicosapentaenoic acid (EPA, C20:5n-3) and docosahexaenoic acid (DHA, C22:6n-3) in human health terms. Numerous clinical and epidemiological studies have correlated these $n-3$ FA with a positive role in infant development (including neuronal, retinal, and immune function), cardiovascular diseases (including reduced incidence of heart disease in adults), cancer, and various mental illnesses including depression, attention-deficit hyperactivity disorder, and dementia (Swanson et al., 2012). The dietary requirement of these FAs, almost certainly due to the fact that humans have a very limited capacity to synthesize them from the essential precursor $\alpha$-linolenic acid (Hossain, 2011), is therefore of paramount importance in human nutrition. In most marine fish, EPA and DHA content rely as well mostly on dietary sources (Sargent et al., 2002) and are essential FAs for normal growth and survival. Our results showed that $S$. fasciata presented overall higher EPA and DHA content in samples of spawning fish (Table 3). At this maturation stage, similar EPA content was found between genders in muscle and liver samples. Still, as a source of dietary EPA and DHA, ovaries and testes of both stages presented highest contents, with spawning females presenting as much as $221 \mathrm{mg}$ EPA/100 g and $140 \mathrm{mg}$ DHA/100 g (Table 3).

Whalen (2009) reported that high ratio of DHA/EPA has an advantageous impact on consumer health and that DHA is more efficient than EPA in reducing the risk of coronary heart diseases. In all cases, the percentage share of DHA in the lipid exceeded that of EPA, resulting in DHA/EPA ratios $>1$ (Table $4 a$ ). The European Food Safety Authority (2010) recommends for the general adult population a dietary intake of $250 \mathrm{mg}$ EPA + DHA per day. Another study of the International Society for the Study of Fatty Acids and Lipids (2004) indicates for cardio health purposes an intake of at least $500 \mathrm{mg}$ EPA + DHA per day. The high levels of EPA + DHA in muscle, liver and gonads of lesser amberjack present it as an important species in human diet and health.

In addition to the high proportions of these $n-3$ FAs, low concentrations of other $n-3$ FA were found, including the essential linolenic acid (C18:3n-3), which was not detected. This finding 
Table 2

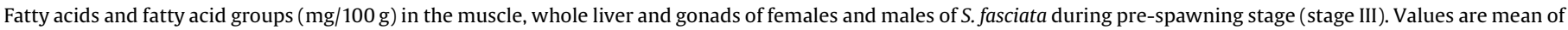
quadruplicates \pm SD; Different letters within the same tissue represent statistical differences between genders $(p<0.05)$.

\begin{tabular}{|c|c|c|c|c|c|c|}
\hline \multirow[t]{2}{*}{ Fatty acids (mg/100 g) } & \multicolumn{2}{|l|}{ Muscle } & \multicolumn{2}{|l|}{ Liver } & \multicolumn{2}{|l|}{ Gonads } \\
\hline & Female & Male & Female & Male & Female & Male \\
\hline \multicolumn{7}{|l|}{ SFA } \\
\hline $\mathrm{C} 14: 0$ & $176 \pm 2.3^{\mathrm{a}}$ & $117 \pm 16.2^{\mathrm{b}}$ & $111 \pm 6.4^{\mathrm{a}}$ & $240 \pm 2.8^{b}$ & $142 \pm 19.4^{\mathrm{a}}$ & $23.8 \pm 2.1^{\mathrm{b}}$ \\
\hline $\mathrm{C} 16: 0$ & $2216 \pm 19.9^{\mathrm{a}}$ & $1246 \pm 123.0^{\mathrm{b}}$ & $5097 \pm 102^{\mathrm{a}}$ & $3139 \pm 14.4^{\mathrm{b}}$ & $2396 \pm 89.2^{\mathrm{a}}$ & $1456 \pm 100^{\mathrm{b}}$ \\
\hline C18:0 & $640 \pm 8.6^{\mathrm{a}}$ & $333 \pm 18.6^{\mathrm{b}}$ & $2551 \pm 195^{\mathrm{a}}$ & $2047 \pm 42.2^{\mathrm{b}}$ & $689 \pm 62.9^{a}$ & $458 \pm 30.2^{\mathrm{b}}$ \\
\hline Others & $124 \pm 14.9^{\mathrm{a}}$ & $49.7 \pm 4.8^{\mathrm{b}}$ & $153.9 \pm 2.9^{\mathrm{a}}$ & $350 \pm 3.8^{b}$ & $140 \pm 5.6^{\mathrm{a}}$ & $82.9 \pm 5.6^{\mathrm{b}}$ \\
\hline \multicolumn{7}{|l|}{ MUFA } \\
\hline $\mathrm{C} 16: 1 n-9$ & $308 \pm 9.7^{a}$ & $116 \pm 12.8^{\mathrm{b}}$ & $364 \pm 21.1^{\mathrm{a}}$ & $483 \pm 3.2^{b}$ & $326 \pm 24.9^{a}$ & $74.3 \pm 7.5^{\mathrm{b}}$ \\
\hline $\mathrm{C} 18: 1 n-9$ & $2197 \pm 42.5^{\mathrm{a}}$ & $937 \pm 63.4^{\mathrm{b}}$ & $5011 \pm 263^{a}$ & $4002 \pm 14.2^{\mathrm{b}}$ & $2907 \pm 127^{\mathrm{a}}$ & $1128 \pm 73.4^{\mathrm{b}}$ \\
\hline $\mathrm{C} 20: 1 n-9$ & $78.0 \pm 2.8^{\mathrm{a}}$ & $16.2 \pm 0.5^{\mathrm{b}}$ & $99.3 \pm 11.9^{a}$ & $462 \pm 3.5^{b}$ & $54.7 \pm 1.2^{\mathrm{a}}$ & $3.8 \pm 0.4^{\mathrm{b}}$ \\
\hline Others & $103 \pm 42.0$ & n.d. & $128 \pm 13.4^{a}$ & $317 \pm 1.6^{\mathrm{b}}$ & $28.0 \pm 0.3^{\mathrm{a}}$ & $28.0 \pm 5.9^{\mathrm{a}}$ \\
\hline \multicolumn{7}{|l|}{ PUFA } \\
\hline$C 18: 2 n-6$ & $33.9 \pm 1.2^{\mathrm{a}}$ & $11.6 \pm 0.8^{\mathrm{b}}$ & $13.4 \pm 0.1^{\mathrm{a}}$ & $72.6 \pm 0.9^{b}$ & $34.0 \pm 3.5^{\mathrm{a}}$ & $19.5 \pm 1.5^{\mathrm{b}}$ \\
\hline$C 18: 4 n-3$ & $1.2 \pm 0.3$ & n.d. & n.d & n.d & n.d & n.d \\
\hline C20:4n-6 (ARA) & $13.3 \pm 1.6^{\mathrm{a}}$ & $7.6 \pm 0.2^{\mathrm{b}}$ & $30.6 \pm 3.0^{\mathrm{a}}$ & $40.8 \pm 1.8^{\mathrm{a}}$ & $63.0 \pm 0.1^{\mathrm{a}}$ & $119 \pm 0.3^{b}$ \\
\hline $\mathrm{C} 20: 4 n-3$ & $3.8 \pm 0.9$ & n.d. & n.d & n.d & $1.1 \pm 0.0^{\mathrm{a}}$ & $1.0 \pm 0.3^{\mathrm{a}}$ \\
\hline $\mathrm{C} 20: 5 n-3$ (EPA) & $62.5 \pm 3.4^{\mathrm{a}}$ & $19.7 \pm 0.0^{\mathrm{b}}$ & $17.7 \pm 1.1^{\mathrm{a}}$ & $64.3 \pm 0.8^{\mathrm{b}}$ & $113 \pm 0.1^{\mathrm{a}}$ & $98.5 \pm 2.1^{\mathrm{b}}$ \\
\hline $\mathrm{C} 22: 5 n-6$ & $12.2 \pm 1.1$ & n.d. & n.d. & $174 \pm 1.5^{\mathrm{b}}$ & $22.7 \pm 6.2^{\mathrm{a}}$ & $29.6 \pm 0.4^{\mathrm{a}}$ \\
\hline$C 22: 5 n-3$ & $50.6 \pm 4.1^{\mathrm{a}}$ & $31.5 \pm 1.1^{\mathrm{b}}$ & n.d. & $41.8 \pm 2.0$ & $43.6 \pm 7.6^{\mathrm{a}}$ & $111 \pm 0.9^{\mathrm{b}}$ \\
\hline C22:6n-3 (DHA) & $493 \pm 1.5^{\mathrm{a}}$ & $343 \pm 10.5^{\mathrm{b}}$ & $281 \pm 24.4^{\mathrm{a}}$ & $393 \pm 10.3^{b}$ & $998 \pm 15.8^{a}$ & $1247 \pm 12.8^{\mathrm{b}}$ \\
\hline Others & $0.6 \pm 0.4$ & n.d. & n.d. & $10.7 \pm 0.7 b$ & n.d. & n.d. \\
\hline Total - SFA & $3156 \pm 1.3^{\mathrm{a}}$ & $1746 \pm 163^{\mathrm{b}}$ & $7912 \pm 300^{\mathrm{a}}$ & $5776 \pm 63.2^{\mathrm{b}}$ & $3368 \pm 177^{\mathrm{a}}$ & $2020 \pm 138^{\mathrm{b}}$ \\
\hline Total - MUFA & $2687 \pm 7.3^{\mathrm{a}}$ & $1069 \pm 75.8^{\mathrm{b}}$ & $5602 \pm 310^{\mathrm{a}}$ & $5263 \pm 22.5^{\mathrm{a}}$ & $3316 \pm 151^{\mathrm{a}}$ & $1234 \pm 74.6^{\mathrm{b}}$ \\
\hline Total - PUFA & $671 \pm 14.6^{\mathrm{a}}$ & $414 \pm 10.5^{\mathrm{b}}$ & $343 \pm 26.4^{\mathrm{a}}$ & $797 \pm 18.0^{\mathrm{b}}$ & $1276 \pm 26.0^{\mathrm{a}}$ & $1626 \pm 13.5^{\mathrm{b}}$ \\
\hline Total fatty acid & $6514 \pm 8.6^{\mathrm{a}}$ & $3229 \pm 228^{b}$ & $13857 \pm 636^{\mathrm{a}}$ & $11836 \pm 104^{\mathrm{b}}$ & $7959 \pm 302^{\mathrm{a}}$ & $4881 \pm 226^{\mathrm{b}}$ \\
\hline$\sum n-3$ & $611 \pm 10.2^{\mathrm{a}}$ & $395 \pm 11.6^{\mathrm{b}}$ & $299 \pm 23.3^{\mathrm{a}}$ & $500 \pm 13.1^{b}$ & $1156 \pm 23.4^{\mathrm{a}}$ & $1457 \pm 11.4^{\mathrm{b}}$ \\
\hline$\sum n-6$ & $59.4 \pm 3.9^{\mathrm{a}}$ & $19.2 \pm 1.1^{\mathrm{b}}$ & $44.0 \pm 3.1^{\mathrm{a}}$ & $287 \pm 4.2^{\mathrm{b}}$ & $120 \pm 2.6^{\mathrm{a}}$ & $169 \pm 2.1^{b}$ \\
\hline$n-3$ HUFA & $610 \pm 9.9^{a}$ & $395 \pm 11.6^{\mathrm{b}}$ & $299 \pm 23.3^{a}$ & $500 \pm 13.1^{\mathrm{b}}$ & $1156 \pm 23.4^{\mathrm{a}}$ & $1457 \pm 11.4^{\mathrm{b}}$ \\
\hline
\end{tabular}

n.d.: not detected; Limit of detection for all fatty acids: $0.1 \mathrm{mg} / 100 \mathrm{~g}$.

Table 3

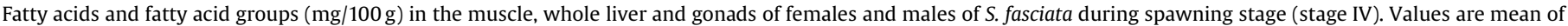
quadruplicates \pm SD; Different letters within the same tissue represent statistical differences between genders $(p<0.05)$.

\begin{tabular}{|c|c|c|c|c|c|c|}
\hline \multirow[t]{2}{*}{ Fatty acids (mg/100 g) } & \multicolumn{2}{|l|}{ Muscle } & \multicolumn{2}{|l|}{ Liver } & \multicolumn{2}{|l|}{ Gonads } \\
\hline & Female & Male & Female & Male & Female & Male \\
\hline \multicolumn{7}{|l|}{ SFA } \\
\hline $\mathrm{C} 14: 0$ & $97.7 \pm 3.1^{\mathrm{a}}$ & $28.7 \pm 0.1^{\mathrm{b}}$ & $102 \pm 0.6^{\mathrm{a}}$ & $79.8 \pm 1.2^{\mathrm{b}}$ & $155 \pm 32.2^{\mathrm{a}}$ & $64.7 \pm 8.3^{\mathrm{b}}$ \\
\hline $\mathrm{C} 16: 0$ & $1080 \pm 5.9^{a}$ & $321 \pm 2.7^{\mathrm{b}}$ & $2709 \pm 47.0^{\mathrm{a}}$ & $1238 \pm 7.9^{\mathrm{b}}$ & $2290 \pm 171.8^{a}$ & $682 \pm 79.5^{\mathrm{b}}$ \\
\hline C18:0 & $322 \pm 1.9^{\mathrm{a}}$ & $120 \pm 0.8^{\mathrm{b}}$ & $1257 \pm 12.0^{\mathrm{a}}$ & $504 \pm 0.1^{b}$ & $610 \pm 71.5^{\mathrm{a}}$ & $286 \pm 20.3^{b}$ \\
\hline Others & $54.0 \pm 3.7^{\mathrm{a}}$ & $29.6 \pm 0.3^{\mathrm{b}}$ & $201 \pm 0.4^{\mathrm{a}}$ & $113 \pm 1.2^{\mathrm{b}}$ & $151 \pm 41.4^{\mathrm{a}}$ & $77.2 \pm 5.5^{\mathrm{a}}$ \\
\hline \multicolumn{7}{|l|}{ MUFA } \\
\hline $\mathrm{C} 16: 1 n-9$ & $125 \pm 10.6^{\mathrm{a}}$ & $45.9 \pm 0.7^{\mathrm{b}}$ & $188 \pm 5.7^{\mathrm{a}}$ & $264 \pm 0.5^{\mathrm{b}}$ & $377 \pm 19.7^{\mathrm{a}}$ & $113 \pm 14.7^{\mathrm{b}}$ \\
\hline $\mathrm{C} 18: 1 n-9$ & $837 \pm 14.6^{\mathrm{a}}$ & $165 \pm 1.2^{\mathrm{b}}$ & $1290 \pm 48.2^{\mathrm{a}}$ & $1305 \pm 2.3^{\mathrm{a}}$ & $2113 \pm 122.9^{\mathrm{a}}$ & $451 \pm 33.9^{\mathrm{b}}$ \\
\hline $\mathrm{C} 20: 1 n-9$ & $25.9 \pm 7.5^{\mathrm{a}}$ & $11.6 \pm 0.2^{\mathrm{a}}$ & $17.3 \pm 1.3^{\mathrm{a}}$ & $54.6 \pm 0.2^{\mathrm{b}}$ & $24.7 \pm 7.2^{\mathrm{a}}$ & $20.4 \pm 0.9^{\mathrm{a}}$ \\
\hline Others & $32.1 \pm 7.7^{\mathrm{a}}$ & $37.8 \pm 1.2^{\mathrm{a}}$ & $112 \pm 5.7^{\mathrm{a}}$ & $176 \pm 0.4^{\mathrm{b}}$ & $53.7 \pm 8.6^{a}$ & $99.0 \pm 0.7^{\mathrm{b}}$ \\
\hline \multicolumn{7}{|l|}{ PUFA } \\
\hline$C 18: 2 n-6$ & $36.9 \pm 6.1^{\mathrm{a}}$ & $20.5 \pm 0.2^{\mathrm{a}}$ & $27.6 \pm 0.1^{\mathrm{a}}$ & $73.3 \pm 3.0^{\mathrm{b}}$ & $82.5 \pm 18.1^{\mathrm{a}}$ & $33.4 \pm 3.9^{\mathrm{a}}$ \\
\hline$C 18: 4 n-3$ & tr. & n.d. & n.d. & n.d. & $2.3 \pm 1.2$ & n.d. \\
\hline C $20: 4 n-6$ (ARA) & $16.9 \pm 3.9^{\mathrm{a}}$ & $23.9 \pm 0.1^{\mathrm{a}}$ & $70.8 \pm 2.3^{\mathrm{a}}$ & $44.9 \pm 0.1^{\mathrm{b}}$ & $114 \pm 21.8^{a}$ & $144 \pm 5.2^{\mathrm{a}}$ \\
\hline $\mathrm{C} 20: 4 n-3$ & $3.3 \pm 2.3$ & n.d. & n.d. & n.d. & $13.5 \pm 2.6$ & n.d. \\
\hline $\mathrm{C} 20: 5 n-3(\mathrm{EPA})$ & $58.3 \pm 6.7^{a}$ & $42.2 \pm 0.0^{\mathrm{a}}$ & $49.2 \pm 5.9^{a}$ & $51.5 \pm 0.2^{\mathrm{a}}$ & $222 \pm 31.4^{\mathrm{a}}$ & $84.7 \pm 3.2^{b}$ \\
\hline$C 22: 5 n-6$ & $17.5 \pm 3.9$ & n.d. & $4.8 \pm 1.1$ & n.d. & $41.2 \pm 8.7$ & n.d. \\
\hline $\mathrm{C} 22: 5 n-3$ & $64.0 \pm 4.3^{\mathrm{a}}$ & $57.2 \pm 0.5^{\mathrm{a}}$ & $16.9 \pm 2.9^{\mathrm{a}}$ & $42.4 \pm 0.7^{\mathrm{b}}$ & $148 \pm 24.7^{\mathrm{a}}$ & $125 \pm 1.0^{\mathrm{a}}$ \\
\hline C22:6n-3 (DHA) & $640 \pm 9.0^{\mathrm{a}}$ & $600 \pm 0.1^{b}$ & $358 \pm 3.7^{\mathrm{a}}$ & $258 \pm 10.6^{\mathrm{b}}$ & $1440 \pm 127^{a}$ & $895 \pm 5.1^{b}$ \\
\hline Others & n.d. & $3.3 \pm 0.1$ & n.d. & $13.8 \pm 0.1^{\mathrm{b}}$ & n.d. & $6.9 \pm 0.3$ \\
\hline Total - SFA & $1553 \pm 3.7^{a}$ & $500 \pm 3.1^{b}$ & $4269 \pm 36.0^{a}$ & $1935 \pm 10.4^{b}$ & $3207 \pm 317^{a}$ & $1110 \pm 114^{b}$ \\
\hline Total - MUFA & $1020 \pm 40.4^{a}$ & $260 \pm 0.8^{b}$ & $1607 \pm 58.3^{a}$ & $1799 \pm 2.3^{b}$ & $2569 \pm 159^{a}$ & $683 \pm 48.8^{b}$ \\
\hline Total - PUFA & $838 \pm 18.9^{a}$ & $747 \pm 0.8^{b}$ & $529 \pm 16.2^{a}$ & $484 \pm 8.8^{b}$ & $2064 \pm 235^{a}$ & $1289 \pm 8.5^{b}$ \\
\hline Total fatty acid & $3411 \pm 63.0^{a}$ & $1506 \pm 4.7^{b}$ & $6405 \pm 78.1^{a}$ & $4218 \pm 3.8^{b}$ & $7839 \pm 711^{a}$ & $3082 \pm 171^{b}$ \\
\hline$\Sigma n-3$ & $766 \pm 4.9^{\mathrm{a}}$ & $699 \pm 0.5^{\mathrm{b}}$ & $425 \pm 12.6^{\mathrm{a}}$ & $353 \pm 11.5^{\mathrm{b}}$ & $1286 \pm 186.5^{\mathrm{a}}$ & $1105 \pm 0.9^{b}$ \\
\hline$\sum n-6$ & $71.3 \pm 14.0^{\mathrm{a}}$ & $47.6 \pm 0.2^{\mathrm{a}}$ & $103 \pm 3.5^{\mathrm{a}}$ & $132 \pm 2.7^{b}$ & $238 \pm 48.6^{\mathrm{a}}$ & $1841 \pm 9.4^{\mathrm{a}}$ \\
\hline$n-3$ HUFA & $766 \pm 4.4^{\mathrm{a}}$ & $699 \pm 0.5^{\mathrm{b}}$ & $425 \pm 12.6^{\mathrm{a}}$ & $353 \pm 11.5^{b}$ & $1823 \pm 185.3^{a}$ & $1105 \pm 0.9^{b}$ \\
\hline
\end{tabular}

n.d.: not detected; Limit of detection for all fatty acids: $0.1 \mathrm{mg} / 100 \mathrm{~g}$ 
Table 4a

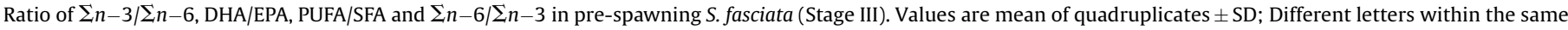
tissue represent statistical differences between genders $(p<0.05)$.

\begin{tabular}{|c|c|c|c|c|c|c|}
\hline & \multicolumn{2}{|l|}{ Muscle } & \multicolumn{2}{|l|}{ Liver } & \multicolumn{2}{|l|}{ Gonads } \\
\hline & Female & Male & Female & Male & Female & Male \\
\hline$\Sigma n-3 / \Sigma n-6$ & $10.3 \pm 0.5^{\mathrm{a}}$ & $20.6 \pm 1.6^{\mathrm{b}}$ & $6.8 \pm 0.1^{\mathrm{a}}$ & $1.7 \pm 0.0^{\mathrm{b}}$ & $9.7 \pm 0.0^{\mathrm{a}}$ & $8.7 \pm 0.0^{\mathrm{b}}$ \\
\hline DHA/EPA & $7.9 \pm 0.4^{\mathrm{a}}$ & $2.6 \pm 0.8^{\mathrm{b}}$ & $16.0 \pm 2.4^{\mathrm{a}}$ & $6.1 \pm 0.1^{b}$ & $8.9 \pm 0.1^{a}$ & $12.7 \pm 0.4^{\mathrm{b}}$ \\
\hline PUFA/SFA & $0.2 \pm 0.0^{\mathrm{a}}$ & $0.2 \pm 0.0^{\mathrm{a}}$ & $0.0 \pm 0.0^{\mathrm{a}}$ & $0.1 \pm 0.0^{\mathrm{b}}$ & $0.4 \pm 0.0^{\mathrm{a}}$ & $0.8 \pm 0.1^{\mathrm{b}}$ \\
\hline$\Sigma n-6 / \Sigma n-3$ & $0.1 \pm 0.0^{\mathrm{a}}$ & $0.1 \pm 0.0^{\mathrm{a}}$ & $0.2 \pm 0.0^{\mathrm{a}}$ & $0.6 \pm 0.0^{\mathrm{b}}$ & $0.1 \pm 0.0^{\mathrm{a}}$ & $0.1 \pm 0.0^{\mathrm{a}}$ \\
\hline
\end{tabular}

Table 4b

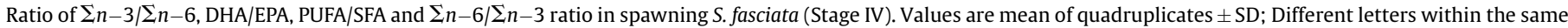
tissue represent statistical differences between genders $(p<0.05)$.

\begin{tabular}{|c|c|c|c|c|c|c|}
\hline & \multicolumn{2}{|l|}{ Muscle } & \multicolumn{2}{|l|}{ Liver } & \multicolumn{2}{|l|}{ Gonads } \\
\hline & Female & Male & Female & Male & Female & Male \\
\hline$\sum n-3 / \sum n-6$ & $11.0 \pm 2.1^{\mathrm{a}}$ & $14.7 \pm 0.1^{\mathrm{a}}$ & $5.4 \pm 1.6^{\mathrm{a}}$ & $2.7 \pm 0.1^{\mathrm{B}}$ & $5.8 \pm 0.5^{\mathrm{a}}$ & $6.0 \pm 0.3^{a}$ \\
\hline DHA/EPA & $11.1 \pm 1.4^{\mathrm{a}}$ & $14.2 \pm 0.0^{\mathrm{b}}$ & $8.6 \pm 1.6^{a}$ & $5.0 \pm 0.2^{\mathrm{B}}$ & $6.6 \pm 1.3^{\mathrm{a}}$ & $10.6 \pm 0.5^{\mathrm{b}}$ \\
\hline PUFA/SFA & $0.3 \pm 0.0^{\mathrm{a}}$ & $1.5 \pm 0.1^{\mathrm{b}}$ & $0.1 \pm 0.0^{\mathrm{a}}$ & $0.3 \pm 0.1^{b}$ & $0.4 \pm 0.0^{\mathrm{a}}$ & $1.2 \pm 0.1^{\mathrm{b}}$ \\
\hline$\sum n-6 / \sum n-3$ & $0.2 \pm 0.1^{\mathrm{a}}$ & $0.1 \pm 0.0^{\mathrm{a}}$ & $0.2 \pm 0.1^{\mathrm{a}}$ & $0.4 \pm 0.0^{\mathrm{b}}$ & $0.2 \pm 0.1^{\mathrm{a}}$ & $0.2 \pm 0.0^{\mathrm{a}}$ \\
\hline
\end{tabular}

reflects the low level of linolenic in natural diets and variations might be related to changes in the nutritional habits of the fish ( Prato and Biandolino, 2012).

In the total n-6 PUFAs family, arachidonic acid (ARA, C20:4n-6) was the most common FA, followed by linoleic acid (LA, C18:2n-6). Highest amounts of ARA were observed in gonads of both maturity stages, varying between 119 and $623 \mathrm{mg} / 100 \mathrm{~g}$ in pre-spawning fish and $114-143 \mathrm{mg} / 100 \mathrm{~g}$ in spawning fish (Tables 2 and 3 ).

Pigott and Tucker (1990) suggested that $\Sigma n-3 / \Sigma n-6$ fatty acid ratio is a useful indicator for comparing relative nutritional value of fish of different species. Generally, fish lipids and fish products have much higher $\Sigma n-3 / \sum n-6$ ratio than is recommended (1:5) (Hossain, 2011). Results of this study showed that S. fasciata contained higher proportions of $n-3$ than of $n-6$ PUFAs resulting in a very beneficial and desirable $\Sigma n-3 / \sum n-6$ ratio (Table $4 a$ ). Correspondingly, the ratios of $\Sigma n-3 / \Sigma n-6$ were lower than the suggested by WHO/FAO (Table 4b), whose recommendation is that, in total daily diet the $\Sigma n-6 / \sum n-3$ ratio should be between $1: 1$ and 5:1 (Simopoulos, 2002).

The Department of Health of the United Kingdom (1994) set a recommended ratio of PUFA/SFA at no less than 1:10. Muscle ratios were higher than recommended, as seen in Tables $4 \mathrm{a}$ and $4 \mathrm{~b}$, indicating the health benefits of this species. Moreover, overall comparison of fatty acid families between the two maturation stages highlights that within each gender, MUFA levels tended to decrease, whereas an increase in PUFA levels was observed.

\section{Conclusion}

Although extensive research exists describing fish lipids and fatty acid composition of many species and its nutritional benefits, there is limited information on variability of these important nutritional parameters according to the fish gender and maturity stage. The results of the present study provide evidence that despite heterogeneity of lipid content among tissues, according to gender and maturity stage, S. fasciata may be classified as a medium or high fat fish. From a nutritional stand point, spawning females are a better source of EPA and DHA, and particular attention must be paid to these FAs content in the ovaries. The proportion $\Sigma n-3 / \Sigma n-6$ indicates that of the three analyzed tissues, muscle has the highest ratio regardless of gender and maturity stage. Likewise, the fact that the proportion of EPA in the muscle is higher than ARA proves this species is a healthy nutritional source. Results also highlight that alternative edible and nutritive parts of fish are available for human consumption. Still, there is the need for nutrition education and more effective communication about fish to the broader public.

\section{Acknowledgements}

This study was partially supported by the Oceanic Observatory of Madeira (M1420-01-0145-FEDER-000001-Observatório Oceânico da Madeira-OOM). The authors would like to thank to Dra. Graça Faria for histological determinations and to Dra. Maria João Aveiro as well as all the technicians of Madeira Fisheries Directorate for their kind collaboration during measurement of meristic characters and determination of histological stage.

\section{References}

Ackman, R.G., 1989. Nutritional composition of fats in sea foods. Prog. Food Nutr. Sci. $13(3-4), 161-289$.

Andaloro, F., Falautano, M., Sinopoli, M., Passarelli, F.M., Pipitone, C., Addis, P., Cau, A., Castriota, L., 2005. The lesser amberjack Seriola fasciata (Perciformes: carangidae) in the Mediterranean: a recent colonist? Cybium 29 (2), 141-145.

Bligh, E.G., Dyer, W.J., 1959. A rapid method of total lipid extraction and purification. Can. J. Biochem. Physiol. 37, 911-917.

Brown-Peterson, N.J., Wyanski, D.M., Saborido-Rey, F., Macewicz, B.J., LowerreBarbieri, S.K., 2011. A standardized terminology for describing reproductive development in fishes. Mar. Coast. Fish. Dyn. Manage. Ecosyst. Sci. 3, 52-70.

Deidun, A., Castriota, L., Arrigo, S., 2011. A tale of two Atlantic fish migrants: records of the lesser amberjack Seriola fasciata and the African hind Cephalopholis taeniops from the Maltese Islands. J. Black Sea/Mediterr. Environ. 17 (3), 223 233.

EFSA Panel on Dietetic Products, Nutrition, and Allergies (NDA), 2010. Scientific opinion on dietary reference values for fats, including saturated fatty acids, polyunsaturated fatty acids, monounsaturated fatty acids, trans fatty acids, and cholesterol. EFSA J. 8 (3), 1461-1568.

Grigorakis, K., 2007. Compositional and organoleptic quality of farmed and wild gilthead sea bream (Sparus aurata) and sea bass (Dicentrarchus labrax) and factors affecting it: a review. Aquaculture 272, 55-75.

Guil-Guerrero, J.L., Venegas-Venegas, E., Rincón-Cervera, M.A., Suaréz, M.D., 2011 Fatty acid profiles of livers from selected marine fish species. J. Food Compos. Anal. 24, 217-222.

Henderson, R.J., Sargent, J.R., Hopkins, C.C.E., 1984. Changes in the content and fatty acid composition of lipid in an isolated population of the capelin, Mallotus villosus: during sexual maturation and spawning. Mar. Biol. 78, 255-263.

Hossain, M.A., 2011. Fish as source of $n-2$ polyunsaturated fatty acids (PUFAs) which one is better-farmed or wild? Ad. J. Food Sci. Technol. 3 (6), 446-455. 
International Society for the Study of Fatty Acids and Lipids (June 2004). Report of the Sub-Committee on Recommendations for Intake of Polyunsaturated Fatty Acids in Healthy Adults. [online] Available at: http://www.issfal.org/newslinks/ resources/publications/PUFAIntakeReccomdFinalReport.pdf (Accessed September, 2014).

Jawad, L., Mtawej, A., Ibrahim, A., Hassan, M., 2015. First record of the lesser amberjack Seriola fasciata (Teleostei: carangidae) in Syrian coasts. Cah. Biol. Mar. 56, 81-84.

Jayasinghe, C., Gotoh, N., Wada, S., 2003. Variation in lipid classes and fatty acid composition of salmon shark (Lamna ditropis) liver with season and gender. Comp. Biochem. Physiol. 134, 287-295.

Jerez, S., Samper, M., Santamaría, F.J., Villamandos, J.E., Cejas, J.R., Felipe, B.C., 2006 Natural spawning of greater amberjack (Seriola dumerili) kept in captivity in the Canary Islands. Aquaculture 252, 199-207.

Kapiris, K., Apostolidis, C., Baldacconi, R., Basusta, N., Bilecenoglu, M., Bitar, G., Bobori, D.C., Boyaci, Y.Ö., Dimitriadis, C., Dujorovic, M., Dulcic, J., Durucan, F., Gerovasileiou, V., GÖkoĞlu, M., Koutsoubas, D., Lefkaditou, E., Lipej, L., Marković, O., Mavrič, B., Özvarol, Y., Pesic, V., Petriki, O., Siapatis, A., Sini, M. Tibullo, D., Tiralongo, F., 2014. New mediterranean marine biodiversity records (April, 2014). Mediterr. Mar. Sci. 15 (1), 198-212.

Luzia, L.A., Sampaio, G.R., Castellucci, C.M.N., Torres, E.A.F.S., 2003. The influence of season on the lipid profiles of five commercially important species of Brazilian fish. Food Chem. 83, 93-97.

Machado, L.F., Barreiros, J.P., 2006. First record of Seriola fasciata (Carangidae) in the Azores, a northernmost occurence in the NE Atlantic. Cybium 30 (1), 77-78.

Mashek, D.G., Wu, C., 2015. MUFA's. Adv. Nutr. 6, 276-277.

Miyazaki, M., Man, W.C., Ntambi, J.M., 2001. Targeted disruption of stearoyl- CoA desaturase 1 gene in mice causes atrophy of sebaceous and meibomian glands and depletion of wax esters in the eyelid. J. Nutr. 131 (9), 2260-2268.

Nogueira, N., Cordeiro, N., Aveiro, M.J., 2013. Chemical composition: fatty acids profile and cholesterol content of commercialized marine fishes captured in Northeastern Atlantic. J. Fish. Sci. 7 (3), 271-286.

Osman, N.H., Suriah, A.R., Law, E.C., 2001. Fatty acid composition and cholesterol content of selected marine fish in Malaysian waters. Food Chem. 73, 55-60.

Otero, P., Pérez, S., Alfonso, A., Vale, C., Rodríguez, P., Gouveia, N.N., Gouveia, N., Delgado, J., Vale, P., Hirama, M., Ishihara, Y., Molgó, J., Botana, L.M., 2010. Firs toxin profile of ciguateric fish in madeira arquipelago (Europe). Anal. Chem. 82 (14), 6032-6039.

Pigott, G.M., Tucker, B.W., 1990. Effects of Technology on Nutrition. Marcel Dekker, Inc, New York, USA.

Prato, E., Biandolino, F., 2012. Total lipid content and fatty acid composition of commercially important fish species from the Mediterranean. Mar. Grande Sea Food Chem. 131, 1233-1239.
Rodriguez-Barreto, D., Jerez, S., Cejas, J.R., Martin, M.V., Acosta, N.G., Bolaños, A. Lorenzo, A., 2012. Comparative study of lipid and fatty acid composition in different tissues of wild and cultured female broodstock of greater amberjack (Seriola dumerili). Aquaculture 360-361, 1-9.

Saito, H., 2012. Lipid characteristics of two subtropical Seriola fishes, Seriola dumerili and Seriola rivoliana: with differences between cultured and wild varieties. Food Chem. 135, 1718-1729.

Sargent, J.R., Tocher, D.R., Bell, J.G., 2002. The lipids, In: Halver, J.E., Hardy, R.W. (Eds.), Fish Nutrition. 3rd ed. Academic Press, San Diego, pp. 181-257.

Sidhu, K.S., 2003. Health benefits and potential risks related to consumption of fish or fish oil. Regul. Toxicol. Pharm. 38 (3), 336-344.

Simopoulos, A.P., 2000. Human requirement for $\mathrm{n}-3$ polyunsaturated fatty acids. Poult. Sci. 79, 961-970.

Simopoulos, A.P., 2002. The importance of the ratio of omega 6/omega 3 essential fatty acids. Biomed. Pharmacother. 56, 365-379.

Smith-Vaniz, W.F., Williams, J.T., Brown, Pina Amargos, F. Curtis, M. (2015) Seriola fasciata. The IUCN Red List of Threatened Species 2015: e.T190139A16510652.

Smith-Vaniz, W.F. 1986. Carangidae. In: Whitehead, P.J.P., Bauchot, M.L., Hureau, J.C., Nielsen, J., Tortonese, E. (Eds.), Fishes of the Northeastern Atlantic and the Mediterranean, Vol. 2. UNESCO, Paris, pp. 815-844.

Smith-Vaniz, W.F., 2002. Carangidae. In: Carpenter, K. (Ed.), The Living Marine Resources of the Western Central Atlantic. Vol. 3: Bony Fishes, Part 2 (Opistognathidae to Molidae, Sea Turtles and Marine Mammals. FAO, Rome, pp. 1426-1460.

Steffens, W., 1997. Effects of variation in essential fatty acids in fish feeds on nutritive value of freshwater fish for humans. Aquaculture 151, 97-119.

Swanson, D., Block, R., Mousa, S.A., 2012. Omega-3 fatty acids EPA and DHA: health benefits throughout life. Adv. Nutr. 3, 1-7.

Thakur, D.P., Morioka, K., Itoh, N., Wada, M., Itoh, Y., 2009. Muscle biochemical constituents of cultured amberjack Seriola dumerili and their influence on raw meat texture. Fish. Sci. 75, 1489-1498.

Department of Health of the United Kingdom, 1994. Nutritional Aspects of Cardiovascular Disease. Report of the Health and Social Subject, vol. 46. Her Majesty's Stationery Office, London.

Tocher, D.R., 2003. Metabolism and functions of lipids and fatty acids in teleost fish. Rev. Fish. Sci. 11 (2), 107-184.

Turchini, G.M., Mentasti, T., Frøyland, L., Orban, E., Caprino, F., Moretti, V.M., Valfre, F., 2003. Effects of alternative dietary lipid sources on performance, tissues chemical composition, mitochondrial fatty oxidation capabilities and sensory characteristics in brown trout (Salmo trutta L.). Aquaculture 225, 251-267.

Whalen, J., 2009. Fishy Business Aquaculture, Omega-3 Fatty Acids and Health. Presented at Aquaculture America, Seattle, Washington. 